\title{
A Modified Homomorphic Filter for Image Enhancement
}

\author{
Gu Hailan \\ College of Textile and Garment, \\ Hebei University of Science and Technology, \\ Shijiazhuang, China \\ E-mail:guhailan@163.com
}

\author{
Lv Wenzhe \\ College of Information Science and Engineering, \\ Hebei University of Science and Technology, \\ Shijiazhuang, China \\ E-mail: lwzhe@hebust.edu.cn
}

\begin{abstract}
In the paper, the homomorphic filter is improved, and a new filter is made, which is based on the low-pass frequency filter and high-pass filter. The performance of the new filter is analysised through comparing to other classical filter. The new filter is experimented, and its application was discussed.
\end{abstract} filter

Keywords-Homomorphic filter, Ringing effect, Butterworth

\section{INTRODUCTION}

In the technique of the digital image processing, an image $f(x, y)$ can be expressed by the product of illuminate function $\mathrm{fi}(\mathrm{x}, \mathrm{y})$ and glint function $\mathrm{fr}(\mathrm{x}, \mathrm{y})$. Illuminate function description scenery of illuminate, have nothing to do with the scenery; Glint function include scenery of detail, with illuminate irrelevant. Because the bright degree of scene illuminate general is slow-moving variety, so the frequency characteristics of illuminate function concentrate on the low frequency segment。 The scenery have more detail and edge, so the frequency characteristics of glint function concentrate on the HF segment. On the other hand, lighting function of describing component changes amplitude and graphical information contained less, but glint function description of scenery, the gray degree class of the picture less but the information be more, have to expand it for this. The homostasis filter is exactly created according to this, it die down low frequency but strengthen the HF segment on the other hand, so can make detail and edge strengthen, the thus more in keeping with person or calculator carry on analysis processing.

Homomorphic filtering processes are shown in Figure 1.

First take the $\log$ of $\mathrm{f}(\mathrm{x}, \mathrm{y})=\mathrm{fi}(\mathrm{x}, \mathrm{y}) \cdot \mathrm{fr}(\mathrm{x}, \mathrm{y})$, then the Fourier transform is, the results multiply $\mathrm{H}(\mathrm{u}, \mathrm{v})$. then the Fourier inverse transform, and the index it, get $g(x, y)$. The Schematic profile of Homostasis filter's Transfer function $\mathrm{H}(\mathrm{u}, \mathrm{v})$ is shown in Figure 2.Rotate it around the vertical axis 360 degrees, get a full two dimensional transfer function $\mathrm{H}(\mathrm{u}, \mathrm{v})$. In Figure $2 \mathrm{TL}<1, \mathrm{Tk}>1$, obviously, the characteristics of low frequency are compressed, but the high frequency expanded.

The image homostasis filter belongs to frequency domain processing category, however it is still on the gray scope adjustments, it is still a grey correction method. According to the characteristics and needs of different image, choose different $\mathrm{H}(\mathrm{u}, \mathrm{v})$, can obtain satisfactory results. We know, noise generally located in high frequency, pure homostasis filter cannot eliminate noise. In addition, in the filtering process of digital image processing, low-pass filter, high-pass filter, or homostasis filter is conducted separately. It can achieve the required filtering effect, but Lack of various filter transfer function parameters used in the overall grasp. In view of this limitation, based on the frequency domain of low-pass filter and high-pass filter, improve the homostasis filter, Put forward a new filter, its performance was analyzed, and apply this filter in the experiment.

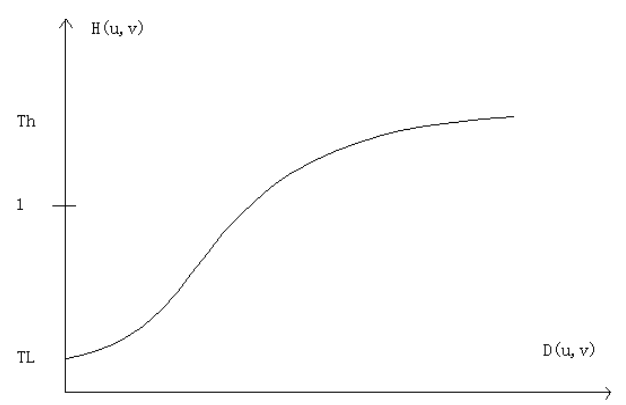

Figure 2. The Schematic profile of Homomorphic filter's Transfer function $\mathrm{H}(\mathrm{u}, \mathrm{v})$

\section{THE IMPROVED HOMOSTASIS FILTER}

The Schematic profile of improved Homomorphic filter's Transfer function H(u,v) is shown in Figure 3.

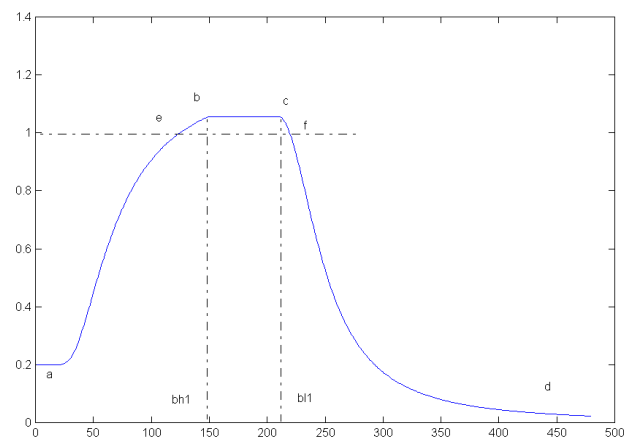

Figure 3. The Schematic profile of improved Homostasis filter 
The improved homostasis filter's Transfer function $\mathrm{H}(\mathrm{u}, \mathrm{v})$ is:

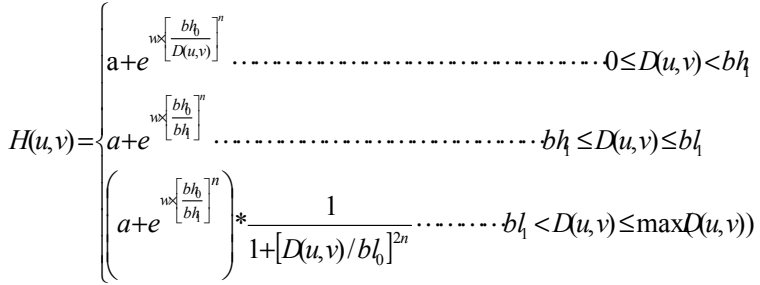

Among this, $\mathrm{n}=1,2,3, \ldots, \mathrm{D}(\mathrm{u}, \mathrm{v})$ expressed the distance from the original point of frequency plane to point $(\mathrm{u}, \mathrm{v})$, $\mathrm{D}(\mathrm{u}, \mathrm{v})=[\mathrm{u} 2+\mathrm{v} 2] 1 / 2$ 。 $\max (\mathrm{D}(\mathrm{u}, \mathrm{v}))$ express the maximum of $\mathrm{D}(\mathrm{u}, \mathrm{v})$. In Figure $3, a-b$ section is the Transfer function of index high-pass filter, which truncation frequency is bh0. Parameter a should be chosen to start from a point e $\mathrm{H}(\mathrm{u}, \mathrm{v})>1 ; b-c$ section is a+exp $(-0.347 \times(\mathrm{bh} 0 / \mathrm{b} 10) \mathrm{n}) ; \mathrm{c}-\mathrm{d}$ section is the transfer function of Butterworth low-pass filter, which truncation frequency is bl0,, Parameter a can control the weakened degree of low frequency, e-f section is should be strengthened frequency band, a-e and f-d section are should be weakened frequency band. The improved Homostasis filter actually is a band-pass, band stop filter.

We know, frequency domain filtering process is realized by selecting the appropriate filter, modified image spectrum. The homostasis filter process is no exception, it is shown in Figure 4. First of all, the original image is the Fourier transform, the transformed results to modify the image spectrum by multiplying $\mathrm{H}(\mathrm{u}, \mathrm{v})$, then take a Fourier inverse transform, get the filtering results $\mathrm{g}(\mathrm{x}, \mathrm{y})$.

\section{FILTER PERFORMANCE ANALYSIS}

Performance evaluation of a filter there are three main, that is," Ringing effect"," Image blur degree (IBD)"and "Smoothing noise effect". "Smoothing noise effect" is aimed at low-pass filter. We compared the improved homostasis filter with the classic ideal low-pass filter, Ladder low pass filter, and Butterworth low-pass filter, compared to the three performance indicators to analyze the performance. The following ideal, Ladder and Butterworth filters are specified as non-specific low-pass filter.

\section{A. Ringing effect}

Figure 5 is Truncation frequency D $0=30$ ideal, Ladder, Butterworth filters, and the improved homostasis filter, comparison of ringing effect. The improved homostasis filter's parameter are: $\mathrm{a}=0.3, \mathrm{bh} 1=40, \mathrm{bh} 0=100$, $\mathrm{b} 11=70, \mathrm{~b} 10=30$, Make $\mathrm{bl}=30$, in order to make The improved homostasis filter with other filter truncation frequency of the same.

The ideal low-pass filter's Transfer function $H(u, v)$, because mutations in the office from 1 to 0 , Therefore, its impulse response manifested in the spatial domain in the form of concentric rings 。 The more concentric rings, indicating that the more severe ringing effect B Butterworth filter has no obvious discontinuity between the pass band and stop band, the number of shock response concentric rings is very small in the space domain, so very light ringing effect. The attenuation of Ladder filter's transfer function between the ideal filter and Butterworth filter with a smooth transition zone. The Ladder filter' shock response concentric rings than ideal filter concentric rings for less, than Butterworth filter concentric rings for more, therefore, the ringing effect of light, than Butterworth filter ringing effect to more serious. The improved filter has no obvious discontinuity between the pass band and stop band also, the number of shock response concentric rings is less than ideal filter and Ladder filter, but a few more than Butterworth filter ,so very light ringing effect.

By contrast, we can conclude: the Improved filter ringing effects is less than ideal filter and ladder filter, but a slightly stronger than Butterworth filter.

\section{B. Image blur degree (IBD)}

Figure 6 is the Image blur degree comparison of Ideal filter, Butterworth filter, and improved filter, among this, the Truncation frequency of ideal filter, Butterworth filter are $\mathrm{D} 0=30$, parameters of the improved filter area $=0.6, \mathrm{bh} 1=20$, $\mathrm{bh} 0=100, \mathrm{bl}=40, \mathrm{~b} 10=30$.

The image blur degree is directly related to the Ringing effect of using filter. Normally, the use of filter ringing effect is stronger, the more fuzzy images. Since the ideal filter attenuation is mutation, so the fuzzy degree of the most serious, Butterworth filter attenuation is relatively smooth, so its very light blur, The improved filter transfer function with the c-d section is of the transfer function for the Butterworth filter, Therefore, the image blurring is also very light.

By comparison, we can conclude: the fuzzy Image of improved filter is very light, with the Butterworth filter is close to the image blurring, but a much lesser extent than the ideal filter image blurring.

\section{C. the smoothing noise effect}

Figure 7 is the smoothing noise effect comparison of the ideal filter, Butterworth filter and the improved homostasis filter . the Truncation frequency of ideal filter, Butterworth filter are $\mathrm{D} 0=30$, parameters of the improved filter area $=0.6, b h 1=20, b h 0=100, b l 1=40, b 10=30$. In order to compare the effect of noise smoothing filter, we have the original image superimposed on a mean of 0.02 , the Gaussian noise variance of 0.005 .

The ideal low-pass filter's Transfer function $\mathrm{H}(\mathrm{u}, \mathrm{v})$, because mutations in the office from 1 to 0 , Thus, in the high frequency noise was completely eliminated,. The Butterworth filter and improved filter transfer function with the state of the tail (high frequency) is a smooth gradient, so that the retention of a portion of high frequency, so the effect of noise smoothing filter for noise smoothing than ideal results to worse.

By comparison, we can conclude that: improved filter with the ordinary noise smoothing effect, and the 
Butterworth filter smoothing effect of noise is very close to, but lower than the ideal filter noise smoothing effect worse.

By ringing effects, image blurring and smoothing effect of noise comparison of the three performance indicators, we have come to the ideal filter, ladder filter, Butterworth filter, and improved filter performance with the state list, shown in Table 1. We can conclude: the improved homostasis filter with the state of Butterworth filter performance is almost the same.

\section{EXPERIMENT AND DISCUSSION}

An image obtained in the experiment, shown in Figure 8, (Experiments using Daheng image acquisition card, SONY Hyper HAD Color camera, image size $426 \times 426$, the original image is superimposed on the mean 0 , variance Gaussian noise 0.02 ), its application results with the improved homostasis filter are shown in Figure 9. The parameters of the improved filter transfer function are, $a=0.7$, bh $1=50$, bh $0=15, b 11=65, b 10=20$.

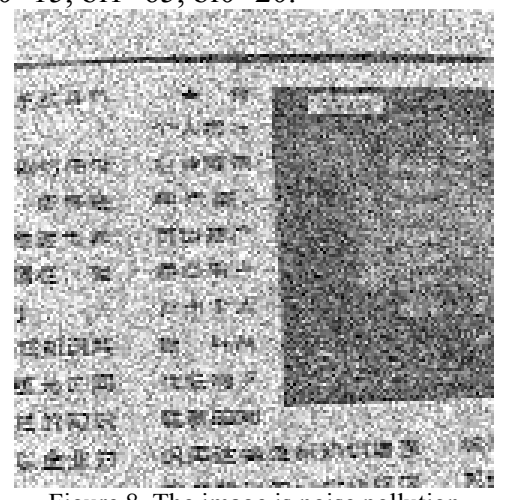

Figure 8 . The image is noise pollution

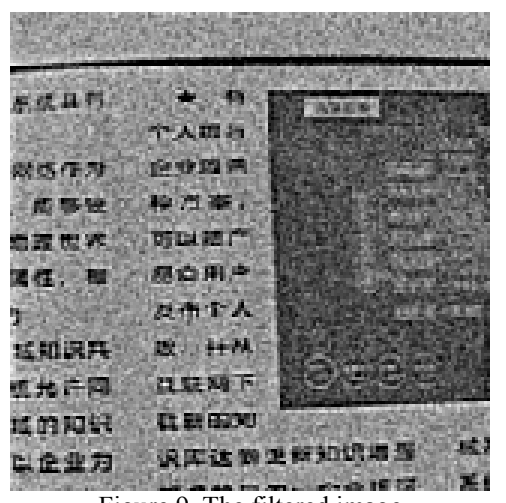

Figure 9 . The filtered image

By comparing images before and after filtering, can be found full of filtering before the image noise, image noise cover edges and details are unclear, the entire image looked very rough。The filtered image, the noise greatly reduced, the details of prominent edge clearer, the whole image looked very fine, visual effects, improved.

In addition, in practice, the a-b section of Figure 3 can be index (or Butterworth) filter transfer function; b-c segment is a constant; c-d section can be index (or Butterworth) filter transfer function. Paragraphs transfer function can be used to select the actual situation. Figure 10 is the expression (2) profile, which, as the index of low-pass filter $\mathrm{a}-\mathrm{b}$ section transfer function; $\mathrm{b}-\mathrm{c}$ segment is a constant; c-d section of the Butterworth high pass filter transfer function.

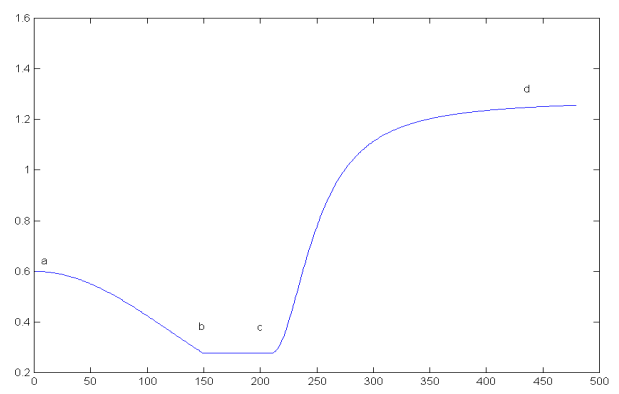

Figure 10. The expression (2) profile

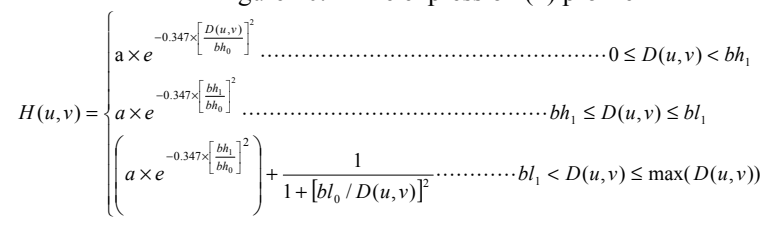

Expression (2) filter expressed by the "ring effect", "image blur degree " and "noise smoothing effect" of thethree performance indicators and expression (1) of the filter expressed almost no difference in performance. Expression (2) the transfer function of the filter that applies to low-frequency be reduced, while strengthening the high-frequency case, it is actually a kind of band pass and band stop filter.

\section{CONCLUSION}

By the three performance indicators comparison of the improved homostasis filter and the classical ideal filter, ladder filter and Butterworth filter, and the improved homostasis filter practical application, We may have to conclusion: the improved performance of the same state filter Butterworth filter with comparable. More prominent, it's the adjustment of parameters is very flexible and can be deformed according to the actual situation, the wider application range. Therefore, the improved filter would be an effective filter.

\section{REFERENCE}

[1] ZHANG Yu-jin. Image Engineering( I )Image Processing(Second Edition) [M],2006, ISBN:7-302-12445-0.

[2] SHEN Ting-zhi. Digital Image Processing and Pattern Recognition[M], 2007, ISBN: 7-810-45403-2.

[3] Rafael C.Gonzalez. Digital Image Processing Using Matlab[M], 2005, ISBN:7-121-01456-4.

[4] WANG Xiao-dan. System Analysis and Design Using Matlab-Image Processing[M], 2000,ISBN:7-560-60920-1.

[5] Ma Long-tian; Zhang Cheng-yi. Optimal design of homorphic filter based on Matlab. Journal of Applied Optics[J], 2010,PP:584-588.

[6] Luo Hai-xia; Liu Bin; Long Yong-hong; et al. Application of Homomorphic Filtering in Illumination Compensation. Journal of Hunan University of Technology[J],2008,PP:23-27. 


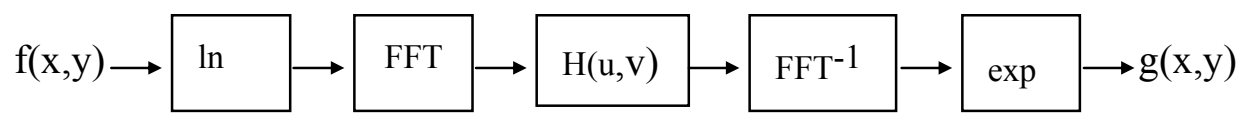

Figure 1. Homomorphic filtering process

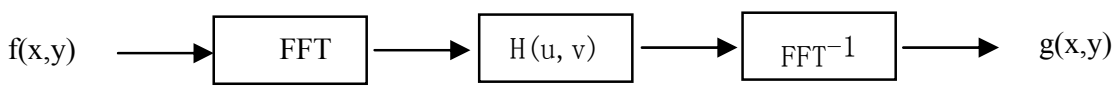

Figure 4. Frequency domain filtering process

the classic ideal low-pass filter

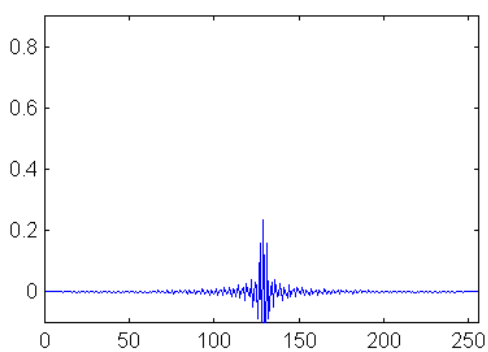

the Butterworth low-pass filter

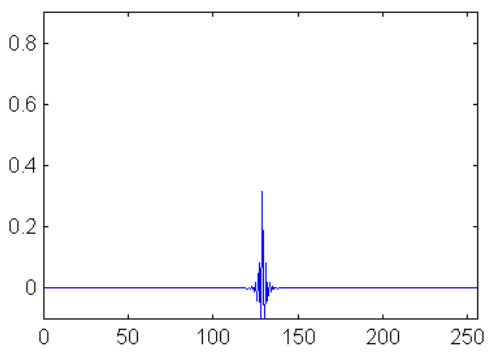

the Ladder low-pass filter

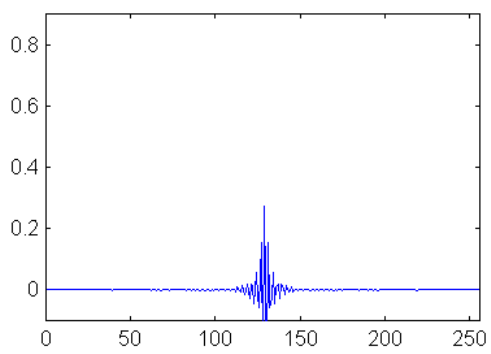

the improved homostasis filter

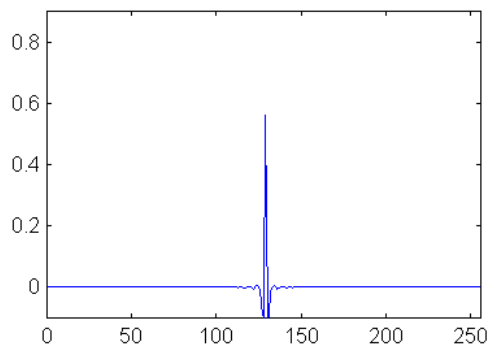

Figure 5. Comparison of Ringing effect

the original image

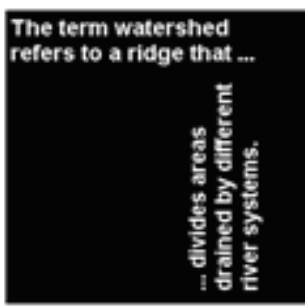

the Butterworth filter

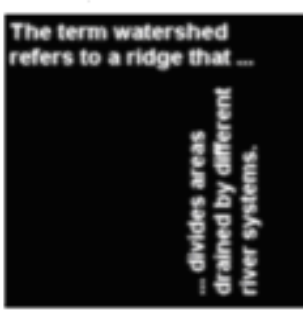

the ideal low-pass filter

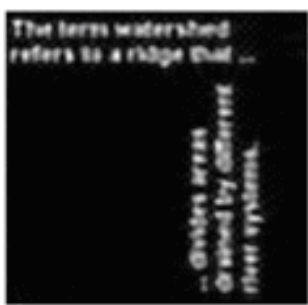

the improved homostasis filter

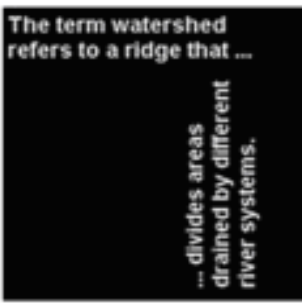

Figure 6. comparison of Image blur degree 
superimposed noise

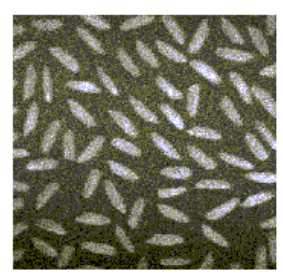

the Butterworth filter

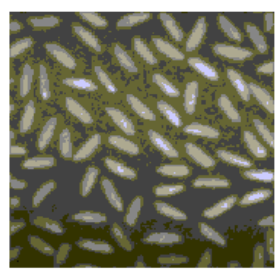

the ideal low-pass filter

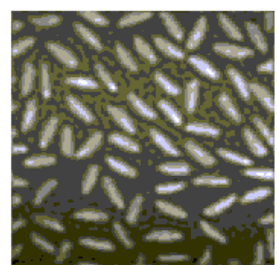

the improved homostasis filter

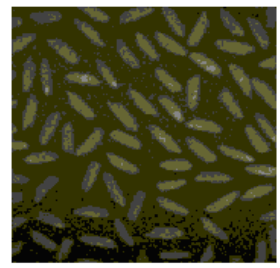

Figure 7. comparison of Smoothing noise effect

TABle I. Performance List

\begin{tabular}{cccc}
\hline Category & Ringing effec & Image blur degree & Smoothing noise effect \\
\hline The ideal low-pass filter & Serious & Serious & The best \\
\hline The Ladder filter & Lighter & Light & Good \\
\hline The Butterworth filter & Immensely light & Very light & ordinary \\
\hline The improved filter & Immensely light & Very light & ordinary \\
\hline
\end{tabular}

